\title{
Characterization of the Dynamic Equilibrium between Closed and Open Forms of the Benzoxaborole Pharmacophore
}

\author{
Sergey Vshyvenko, ${ }^{\dagger}$ Marissa L. Clapson, Itaru Suzuki, ${ }^{\ddagger}$ and Dennis G. Hall* \\ Department of Chemistry, 4-010 Centennial Centre for Interdisciplinary Science, University of Alberta, Edmonton, Alberta T6G 2G2, \\ Canada
}

\section{Supporting Information}

ABSTRACT: Benzoxaboroles are a class of five-membered hemiboronic acids that recently attracted significant attention as a new pharmacophore on account of their unique structural and physicochemical properties and their ability to interact selectively with biomolecules. Their structural behavior in water and its effect on their physiological properties remain unclear, especially the question of dynamic hydrolytic equilibrium of the oxaborole ring. Herein, we used NMR spectroscopy, in mixed aqueous-organic solvent, to confirm the strong preference for the closed form of benzoxaborole and its six- and seven-membered homologues over the open boronic acid form. Only with the eight-membered homologue does the cyclic form become unfavorable. Using dynamic VT-NMR studies with designed probe compound 20, we demonstrate that the oxaborole ring undergoes rapid hydrolytic ring closing-opening at ambient temperature at a rate of $>100 \mathrm{~Hz}$ via a mechanism featuring rate-limiting protontransfer steps. This knowledge can help provide a better understanding of the behavior of benzoxaboroles in biological systems.

KEYWORDS: Benzoxaborole, boron heterocycles, hydrolysis, ring size, VT-NMR
$\mathrm{T}^{\mathrm{s}}$ he benzoxaborole ring system has emerged as a promising pharmacophore on the strength of the recent approval of the antifungal drug tavaborole $(2, \text { AN2690 })^{1}$ and the potency demonstrated by numerous derivatives against an impressive diversity of target classes (Figure 1). ${ }^{2-8}$ The parent compound,

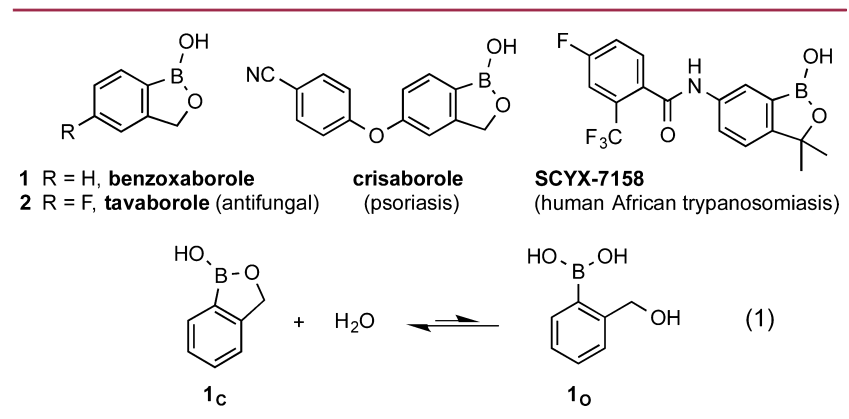

Figure 1. Benzoxaborole (1), derivatives of pharmaceutical interest and its two possible forms in water (eq 1).

benzoxaborole (1), was first prepared by Torssell in $1957 .^{9}$ It was described as a highly water-soluble and unusually robust boronic acid derivative with great resistance to both acidic and basic aqueous deboronation. At the time, its structure was proposed to be the cyclic monodehydrated form on the grounds of elemental analysis ${ }^{9}$ and ebullioscopic molecular weight determination. ${ }^{10}$ Although the asserted preference for a cyclic hemiboronic structure $\mathbf{1}_{\mathrm{C}}$ over the open form $\mathbf{1}_{\mathrm{O}}$ (Figure 1 , eq 1 ) is reasonable based on the lability of $\mathrm{B}-\mathrm{O}$ bonds and the stability of five-membered rings, until this day there appears to exist no published experimental evidence for it aside from mass spectrometry (in the gas phase) and X-ray crystallography (in thesolid form). ${ }^{11}$ Surprisingly, the structural behavior of $\mathbf{1}$ in aqueous solutions has never been studied systematically.

While studying the chemistry of benzoxaborole (1), Snyder remarked that it "is remarkably resistant to hydrolysis of the link between the alkoxyl group and the boron atom". ${ }^{10}$ Indeed, we are aware of only two instances where a benzoxaborole derivative was observed in its open form: a specific case involving a stable trifluoroborate derivative that is less prone to cyclize due to the strength of $\mathrm{B}-\mathrm{F}$ bonds, ${ }^{12}$ and the peculiar $7-$ hydroxymethylated derivative of $\mathbf{1}$ whose cyclization would lead to a disfavored [5.5] system. ${ }^{13}$ Owing to Le Châtelier's Principle, boronic ester formation is intrinsically unfavored in water. Because the balance of bond enthalpy is neutral $(\mathrm{O}-\mathrm{H}$ and $\mathrm{B}-\mathrm{O}$ bonds are broken to form similar ones), thermodynamically the process of eq 1 is driven solely by the enthalpy of ring formation. This factor, along with the entropic benefit from the release of a water molecule, are the reasons for the asserted preference for the cyclic, closed form of benzoxaborole $\left(\mathbf{1}_{\mathrm{C}}\right.$, Figure 1).

Despite the growing role of benzoxaborole derivatives in pharmaceutical and materials chemistry applications, ${ }^{4-8}$ the important questions of their structure in aqueous solutions (closed or open?) and the possible existence of a dynamic equilibrium between these forms have not been examined in detail. ${ }^{14}$ This knowledge is important in order to gain a better understanding of the physicochemical properties of benzoxaboroles and their behavior in biological systems such as their

Received: August 3, 2016

Accepted: September 21, 2016

Published: September 21, 2016 
transport and pharmacokinetics. Herein, using NMR spectroscopy, we attempt to study the effect of ring size of boronic acid hemiesters $\mathbf{1}$ and $\mathbf{3 - 5}$ on the structure and stability of these compounds in aqueous organic solutions (see Figure 2). Our

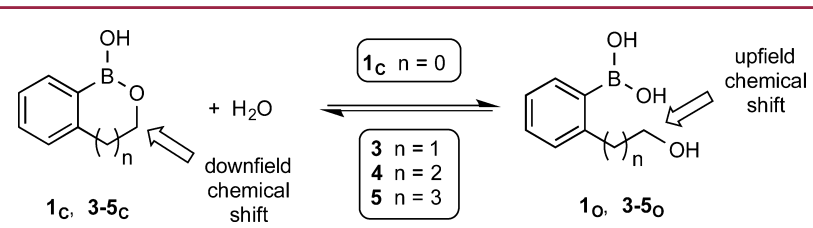

Figure 2. Equilibrium between closed and open form of benzoxaborole homologues up to eight-atom ring size.

initial assumption was that if a closing-opening equilibrium exists, it can be monitored on the time scale of NMR spectroscopy due to sufficient difference between both forms for the chemical shifts of the methylene groups adjacent to the oxygen atom. Thus, when the free alcohol closes into a hemiester with the nearby boronic acid, the electron-withdrawing effect of $\mathrm{B}-\mathrm{O}$ conjugation is expected to move the $\mathrm{CH}_{2} \mathrm{O}$ shift downfield. To substantiate this notion, the analogous methyl ethers with a similar chemical structure, but lacking the ability to cyclize into a hemiboronic ester, were employed as NMR standards.

While some of the targeted compounds were commercially available, others were prepared according to standard synthetic protocols for the synthesis of organoboronic acids. To this end, commercial 2'-bromobenzyl bromide (6) was chosen as a universal substrate in a divergent route to all oxaborole homologues and NMR standards. From 6, the desired alkene intermediates 7 or $\mathbf{8}$ were produced by the corresponding vinylation $^{15}$ or allylation, ${ }^{16}$ followed by a hydroboration/ oxidation step. Either a methylation of the resulting alcohol or installation of a tetrahydropyranyl ether led to all four bromoarenes 11-14. The final step consisted in a standard lithiation/borylation, which after hydrolysis yielded boronic acids 4, 5, 15, and 16 (see Scheme 1).

\section{Scheme 1. Preparation of NMR Probe Compounds}

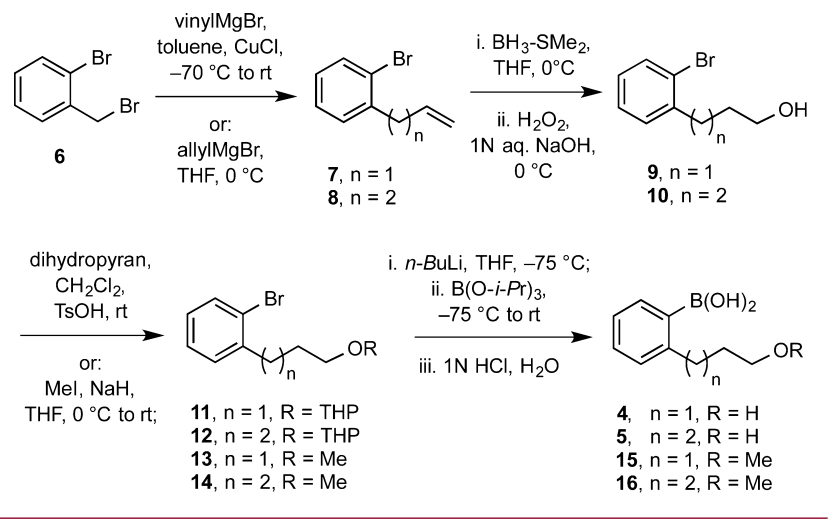

Proton NMR analysis of the parent benzoxaborole $\mathbf{1}$ and all the higher homologues 3-5 was achieved in acetone- $d_{6}: \mathrm{D}_{2} \mathrm{O}$ (9:1) mixture, necessary for compound solubility, at room temperature $\left(293 \mathrm{~K}=20{ }^{\circ} \mathrm{C}\right)$. It was deemed important to perform this analysis in the presence of excess water in order to approach the conditions of physiological systems. On the basis of the large chemical shift differences of $0.4-0.5 \mathrm{ppm}$ for the $-\mathrm{CH}_{2} \mathrm{O}-$ resonance between these compounds and the corresponding control methyl ethers, it can be deduced that only the closed forms of $\mathbf{1}, \mathbf{3}$, and 4 are present at room temperature (Table 1).

Table 1. ${ }^{1} \mathrm{H}$ NMR Shift of Methyleneoxy for Benzoxaborole, its Homologues, and Control Compounds

\begin{tabular}{|c|c|c|c|c|}
\hline entry & compound & $\begin{array}{l}\delta \quad \mathrm{CH}_{2} \mathrm{O} \\
(\mathrm{ppm})\end{array}$ & compound & $\begin{array}{l}\delta \quad \mathrm{CH}_{2} \mathrm{O} \\
(\mathrm{ppm})\end{array}$ \\
\hline 1 & $\mathrm{OH}$ & 4.95 & 17 & 4.55 \\
\hline 2 & ${ }^{3} \mathrm{c}$ & 4.06 & H & 3.58 \\
\hline 3 & $4_{\mathrm{c}}$ & 3.75 & 15 & 3.29 \\
\hline 4 & $\mathrm{OH}_{1}$ & 3.46 & $\mathrm{OH}_{1}$ & 3.29 \\
\hline 5 & $\begin{array}{l}\text { 19a } n=1 \\
19 b n=2 \\
19 c n=3\end{array}$ & $\begin{array}{ll}19 a & 3.68 \\
19 b & 3.51 \\
19 c & 3.48\end{array}$ & & \\
\hline
\end{tabular}

${ }^{a}$ NMR studies were performed in acetone- $d_{6} / \mathrm{D}_{2} \mathrm{O} 9: 1$ mixture at 300 $\mathrm{K}$.

In contrast, the spectrum of $\mathbf{5}$ showed the presence of only the open form $5_{\mathrm{O}}$, with the upfield shift characteristic of the methylene group of control compounds 16 and 19c. This analysis is consistent with basic principles of thermodynamic stability for small and medium rings, where a very large preference for the cyclic forms exists in the case of benzoxaborole (1) and its six-membered homologue (3). The eight-membered medium-ring homologue 5 exists exclusively in its open form, $\mathbf{5}_{\mathrm{O}}$, which dominates because of the less favorable thermodynamics of eight-membered rings and the entropic benefits provided by the additional degrees of freedom of several rotatable bonds on the flexible butanol chain.

To assess the proportions of closed and open forms and thermodynamics of this process, VT experiments were performed. It was found that compounds 1, 3, and 5 remained unchanged in the entire range of temperatures from 203 to 303 $\mathrm{K}$. In contrast, the seven-membered ring compound 4 was found to exist in a mixture of both forms in proportions that varied depending on the temperature. Quite unexpectedly, the amount of the open form, boronic acid $\mathbf{4}_{\mathrm{O}}$, which is almost inexistent at $303 \mathrm{~K}$, increased upon lowering the temperature (see Figure 3). At $203 \mathrm{~K}\left(-70{ }^{\circ} \mathrm{C}\right)$, the open form accounts for about one-third of the mixture (see Supporting Information, Table S2). This outcome is consistent with the notion that two different entropic factors are competing in this equilibrium: (1) the release of a water molecule accompanying the cyclization to the closed form, thus increasing the overall entropy of the 


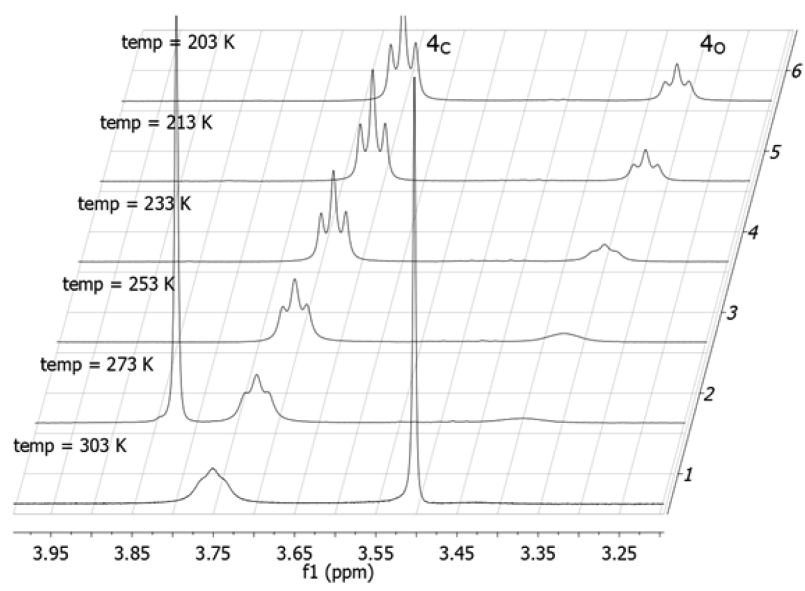

Figure 3. VT studies of compound 4 in acetone $/ \mathrm{D}_{2} \mathrm{O}$ solution showing the temperature dependence of ratio $\mathbf{4}_{\mathrm{O}} / \mathbf{4}_{\mathrm{C}}$.

system vs (2) the entropic benefits from the degrees of freedom of many rotatable bonds in the flexible alkanol side chain of open forms, especially in the higher homologues $\mathbf{4}_{\mathrm{O}}$ and $\mathbf{5}_{\mathrm{O}}$ (see Figure 4). We believe that at the lower temperatures, the

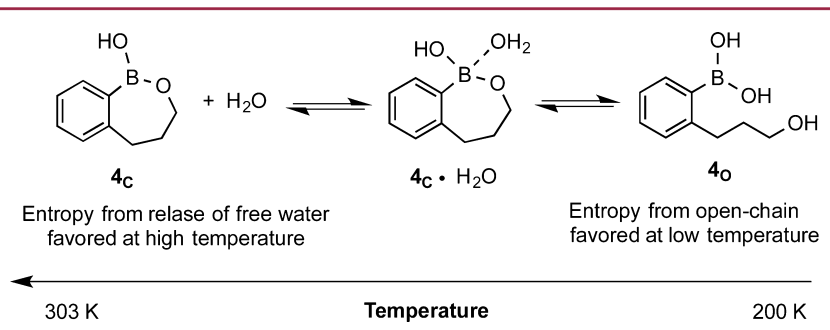

Figure 4. Proposed behavior of seven-membered compound 4 at different temperatures.

release of water in the cyclization does not significantly increase the entropy of the system owing to the more structured character of water and its stronger coordination to the boronic hemiester. The water molecule may remain closely associated with the cyclic compound (e.g., $\mathbf{4}_{\mathrm{C}} \cdot \mathrm{H}_{2} \mathrm{O}$ ) at these low temperatures. The $\mathbf{4}_{\mathrm{O}}: \mathbf{4}_{\mathrm{C}}$ ratio obtained at different temperatures allowed a determination of the free energy of this process $\Delta G_{\mathrm{O} / \mathrm{C}}=-2.6 \mathrm{~kJ} / \mathrm{mol}$ (see Supporting Information).

The above measurements characterize the steady state of an equilibrium occurring between two different forms; the dynamic parameters of the equilibrium cannot be assessed by means of these probes. Consequently, our next objective was to design a DNMR probe molecule to gain direct evidence of a dynamic, reversible hydrolytic equilibrium between the closed and open forms of 1 via the breaking and reclosing of the endocyclic $\mathrm{B}-\mathrm{O}$ bond in aqueous solution. Although several possible probes were considered, we elected to target the unsymmetrical diboronic acid $\mathbf{2 0}$ containing a single secondary benzylic alcohol flanked by two distinct arylboronic acid units (see Figure 5). It was anticipated that probe $\mathbf{2 0}$ would display a dynamic behavior similar to that of benzoxaborole 1 , and therefore it may establish an equilibrium between two isomeric benzoxaboroles $20 \mathrm{a}$ and $20 \mathrm{~b}$ that could be monitored by means of ${ }^{19} \mathrm{~F}$ NMR.

The synthesis of compound 20 began with a selective metalation $^{17}$ of 2-bromoiodobenzene (21), followed by electrophilic quenching with 2-bromo-5-fluorobenzaldehyde (22) (Scheme 2). The resulting alcohol 23 was doubly<smiles>OB1OC(c2ccccc2[18OH])c2ccc(F)cc21</smiles>

$20 \mathrm{a}$<smiles>OB1OC(c2ccc(F)cc2Br)c2ccccc21</smiles>

$20 \mathrm{~b}$
Figure 5. Possible equilibrium between two isomeric forms of diboronic acid $\mathbf{2 0}$ in aqueous solution.

Scheme 2. Preparation of Dynamic NMR Probe 20

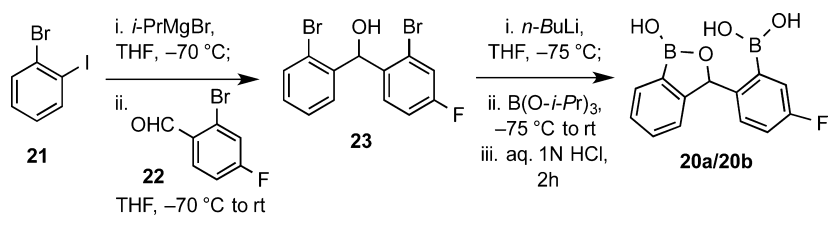

metalated and added to tris(isopropyl)borate. The desired product $\mathbf{2 0}$ was isolated by column chromatography and submitted to VT-NMR studies. Variable temperature experiments were performed in both $90 \%$ acetone $/ 10 \% \mathrm{H}_{2} \mathrm{O}$ and $90 \%$ acetone $/ 10 \% \mathrm{D}_{2} \mathrm{O}$ solutions in order to calculate the equilibrium constants at different temperatures and attempt to estimate the equilibrium isotopic effect. Our initial experiments showed that equilibrium is established rapidly in both systems at different temperatures ranging from ambient to $-70{ }^{\circ} \mathrm{C}$. The VT profile was performed under several increments (213-303 $\mathrm{K})$ at $400 \mathrm{MHz}$ frequency under dilute conditions $(0.06 \mathrm{M})$ to help prevent unwanted aggregation of boronic acid 20. At low, precoalescence temperature, the ${ }^{19} \mathrm{~F}$ NMR spectra show two separate forms of the probe compound in uneven proportions (Figure 6). Upon heating, peak broadening occurs with

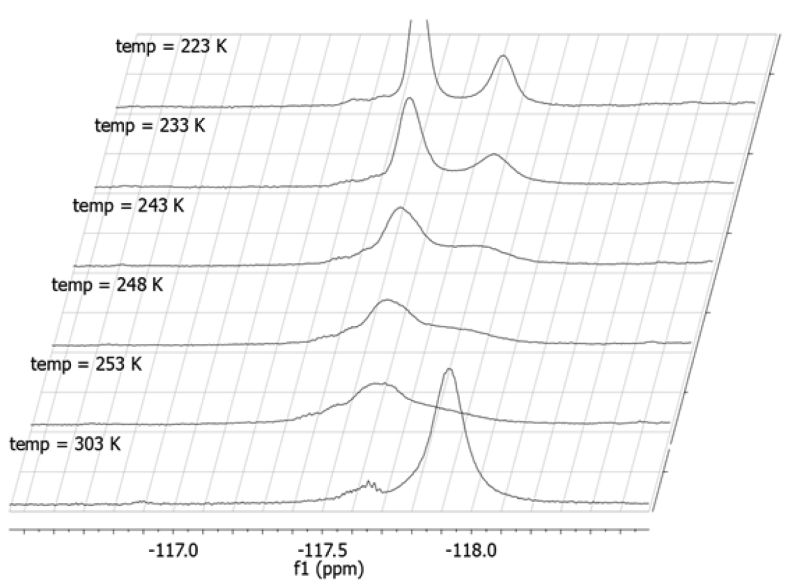

Figure 6. VT equilibrium of 20 in acetone $-\mathrm{H}_{2} \mathrm{O}(9: 1)$.

eventual coalescence into a single resonance. Expectedly, a significant temperature-dependent drift of the ${ }^{19} \mathrm{~F}$ chemical shift occurred, ${ }^{18}$ which complicated the accurate determination of the coalescence temperature $\left(T_{\mathrm{C}}\right)$. DNMR analysis for the interconversion of unequally populated equilibrating species is much less common compared to degenerate processes. However, rate constants of direct and reverse reactions, $k_{\mathrm{a}}$ (for 20a to 20b) and $k_{\mathrm{b}}$ (for 20b to 20a) and equilibrium constants can be estimated before coalescence by utilizing the modified Gutowsky-Holm equation from half-height peak widths, h, for both forms 20a and 20b. ${ }^{19,20}$ The resulting data indicates that the exchange between the two isomeric forms 
occurs in the $\mathrm{D}_{2} \mathrm{O}$ solvent mixture at a rate up to six times slower compared to that observed in $\mathrm{H}_{2} \mathrm{O}$ at comparable temperatures (see Supporting Information, Tables S2 and S3). This behavior can also be deduced by the respective coalescence temperatures of $\sim 290 \mathrm{~K}$ and $\sim 255 \mathrm{~K}$, thus confirming that the $\mathrm{D}_{2} \mathrm{O}$ system requires a higher temperature to reach a fast equilibrium. Overall, these results suggest that a proton-transfer step with at least one molecule of water is involved in the exchange between 20a and 20b (see Figure 7).

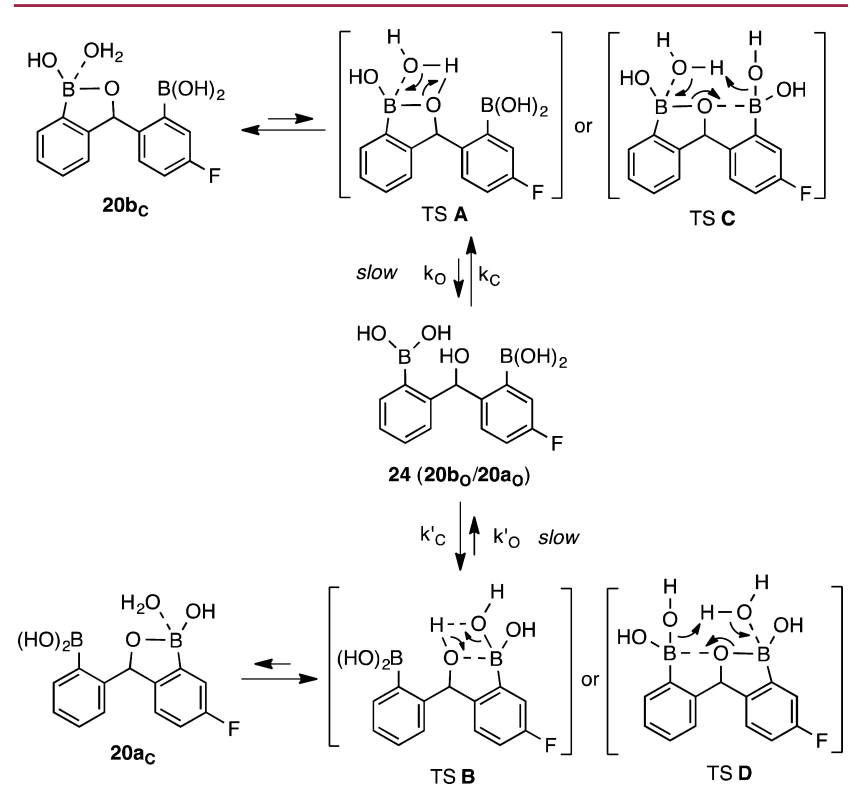

Figure 7. Suggested hydrolytic mechanism and structures of transition states between the two isomeric forms of $\mathbf{2 0}$.

Analysis of the data using the modified Gutowsky-Holm equations allows an estimation of the interconversion rate (see Supporting Information). At low temperatures (e.g., $223 \mathrm{~K}$ ) where isomers are distinguishable, equilibrium in acetone $-\mathrm{D}_{2} \mathrm{O}$ is slow. In the acetone $-\mathrm{H}_{2} \mathrm{O}$ mixture, however, a relatively faster exchange occurs with a frequency around $30 \mathrm{~Hz}$. Close to coalescence temperature, exchange constants reach 111 and 93 $\mathrm{Hz}$, respectively. At room temperature, equilibrium in acetone$\mathrm{H}_{2} \mathrm{O}$ occurs as fast as $135 \mathrm{~Hz}$.

Altogether, these results provide a semiquantitative characterization of the dynamic equilibrium between the open and closed forms of benzoxaboroles, leading to the proposed mechanistic scheme depicted in Figure 7. Recent evidence suggests that boronic esters hydrolyze faster at neutral $\mathrm{pH}$ in their trigonal, neutral form compared to the corresponding hydroxy-boronate anionic form. ${ }^{21}$ Thus, water coordination in neutral $\mathbf{2 0 b _ { C }}$ is followed by the proton transfer step depicted in $\mathrm{TS}_{\mathrm{A}}$, leading to ring opening and formation of diboronic acid 24 (i.e., $20 b_{\mathrm{O}} / \mathbf{2 0 a _ { \mathrm { O } }}$ ). Because ring opening is anticipated to be much slower than ring closure $\left(k_{\mathrm{C}}>k_{\mathrm{O}} ; k_{\mathrm{C}}^{\prime}>k_{\mathrm{O}}^{\prime}\right)$, open intermediate 24 is anticipated to exist in minute concentration and is not observable by NMR. Fast ring closure of $\mathbf{2 4}$ may occur by reversion to $20 b_{C}$ or alternatively toward the other isomer $\mathbf{2 0 a _ { C }}$ via $T S_{B}$. Because ring opening by water is the ratedetermining step of the overall equilibrium interconversion, a strong isotopic retardation effect can be observed in $\mathrm{D}_{2} \mathrm{O}$ cosolvent. It is important to note that although noncooperativity between both boronyl units was assumed, it cannot be ruled out. Though it may be geometrically difficult, intramolecular six-atom hydrolytic pathways may occur via transition states $\mathrm{TS}_{\mathrm{C}}$ and $\mathrm{TS}_{\mathrm{D}}$. Relative to benzoxaborole (1), it is also possible that the secondary alcohol of $\mathbf{2 4}$ benefits from a favorable Thorpe-Ingold effect to give a somewhat inflated closure rate constant. Finally, as demonstrated by the uneven peak integrations at precoalescence temperatures, one of the two isomers exists in slightly higher proportions, which is explained presumably by the differential Lewis acidity of the two boronyl units (the presence of a meta-fluoride lowers the $\mathrm{p} K_{\mathrm{a}}$ of phenylboronic acid by approximately one unit ${ }^{22}$ ). Regardless of its distinct characteristics, we believe that probe compound $\mathbf{2 0}$ provides a reasonable representation of the closing-opening hydrolytic equilibrium of benzoxaborole (1).

In summary, this study confirms the strong preference for the closed form of benzoxaborole (1) and its six- and sevenmembered homologues over the open boronic acid form in aqueous-organic solvent at ambient temperature. Only with the eight-membered homologue does the cyclic form become unfavorable. Surprisingly, the seven-membered homologue showed an increasingly high proportion of the open form at lower temperatures, a trend that can be ascribed to the more organized structure of water and its tighter coordination to the boron atom, leading to a diminished entropic preference toward the cyclization. It was also demonstrated that the closing-opening equilibrium can be monitored by NMR spectroscopy at low temperatures and previously unattainable parameters can be extracted. A probe molecule, diboronic acid 20, was designed for studying the dynamics of the hydrolytic equilibrium between the closed and open forms. It was shown that the exchange between the two isomeric closed forms of diboronic acid $\mathbf{2 0}$ occurs with a significant frequency even at low temperatures. Furthermore, a strong isotopic retardation effect was observed with deuterated water, thus confirming the participation of water and proton transfer steps in the transition state of interconversion. Overall, this study into the structural behavior of benzoxaboroles and its homologues in aqueousorganic solution provides a deeper understanding of this new and important pharmacophore. Insofar as probe $\mathbf{2 0}$ accurately reflects the behavior of benzoxaborole (1), reversible hydrolysis of the oxaborole ring occurs rapidly $(>100 \mathrm{~Hz})$ under physiological conditions.

\section{ASSOCIATED CONTENT}

\section{S Supporting Information}

The Supporting Information is available free of charge on the ACS Publications website at DOI: 10.1021/acsmedchemlett.6b00300.

Full experimental details and compound characterization data, NMR spectral reproductions (PDF)

\section{AUTHOR INFORMATION}

\section{Corresponding Author}

*Phone: 780-492-3141. Fax: 780-492-8231. E-mail: dennis. hall@ualberta.ca.

\section{Present Addresses}

${ }^{\dagger}$ For S.V.: Gilead Alberta ULC, 1021 Hayter Road NW, Edmonton, Alberta T6S 1A1, Canada.

${ }^{\ddagger}$ For I.S.: Osaka University, Research Center for Environmental Preservation, 2-4 Yamadaoka, Suita, Osaka 565-0871, Japan. 


\section{Author Contributions}

The manuscript was written through contributions of all authors. All authors have given approval to the final version of the manuscript.

\section{Notes}

The authors declare no competing financial interest.

\section{ACKNOWLEDGMENTS}

This research was generously funded by the Natural Science and Engineering Research Council of Canada (Discovery Grant 203287-2012 to D.G.H.) and the University of Alberta. We thank Mr. Mark Miskolzie for help with VT-NMR studies.

\section{ABBREVIATIONS}

DNMR, dynamic NMR; NMR, nuclear magnetic resonance; TS, transition structure; VT-NMR, variable temperature NMR

\section{REFERENCES}

(1) Baker, S. J.; Zhang, Y.-K.; Akama, T.; Lau, A.; Zhou, H.; Hernandez, V.; Mao, W.; Alley, M. R. K.; Sanders, V.; Plattner, J. J. Discovery of a New Boron-containing Antifungal Agent, 5-Fluoro-1,3dihydro-1-hydroxy-2,1-benzoxaborole (AN2690), for the Potential Treatment of Onychomycosis. J. Med. Chem. 2006, 49 (15), 44474450.

(2) Akama, T.; Baker, S. J.; Zhang, Y. K.; Hernandez, V.; Zhou, S.; Sanders, V.; Freund, Y.; Kimura, R.; Maples, K. R.; Plattner, J. Discovery and Structure-Activity Study of a Novel Benzoxaborole Anti-Inflammatory Agent (AN2728) for the Potential Topical Treatment of Psoriasis and Atopic Dermatitis. Bioorg. Med. Chem. Lett. 2009, 19 (8), 2129-2132.

(3) Jacobs, R. T.; Plattner, J. J.; Nare, B.; Wring, S. A.; Chen, D.; Freund, Y.; Gaukel, E. G.; Orr, M. D.; Perales, J. B.; Jenks, M.; Noe, R. A.; Sligar, J. M.; Zhang, Y.-K.; Bacchi, C. J.; Yarlett, N.; Don, R. Benzoxaboroles: a New Class of Potential Drugs for Human African Trypanosomiasis. Future Med. Chem. 2011, 3 (10), 1259-1278.

(4) Adamczyk-Woźniak, A.; Cyrański, M. K.; Żubrowska, A.; Sporzyński, A. Benzoxaboroles - Old Compounds with New Applications. J. Organomet. Chem. 2009, 694 (22), 3533-3541.

(5) Baker, S. J.; Tomsho, J. W.; Benkovic, S. J. Boron-Containing Inhibitors of Synthetases. Chem. Soc. Rev. 2011, 40 (8), 4279-4285.

(6) Zhang, J.; Zhu, M.; Lin, Y.; Zhou, H. The Synthesis of Benzoxaboroles and Their Applications in Medicinal Chemistry. Sci. China: Chem. 2013, 56 (10), 1372-1381.

(7) Liu, C. T.; Tomsho, J. W.; Benkovic, S. J. The Unique Chemistry of Benzoxaboroles: Current and Emerging Applications in Biotechnology and Therapeutic Treatments. Bioorg. Med. Chem. 2014, 22 (16), $4462-4473$.

(8) Adamczyk-Woźniak, A.; Borys, K. M.; Sporzyński, A. Recent Developments in the Chemistry and Biological Applications of Benzoxaboroles. Chem. Rev. 2015, 115 (11), 5224-5247.

(9) Torssell, K. Zur Kenntnis Der Arylborsauren 0.3. Bromierung Der Tolyborsauren Nach Wohl-Ziegler. Ark. Kemi 1957, 10, 507-511.

(10) Snyder, H. R.; Reedy, A. J.; Lennarz, W. J. Synthesis of Aromatic Boronic Acids - Aldehydo Boronic Acids and a Boronic Acid Analog of Tyrosine. J. Am. Chem. Soc. 1958, 80 (4), 835-838.

(11) Zhdankin, V. V.; Persichini, P. J., III; Zhang, L.; Fix, S.; Kiprof, P. Synthesis and Structure of Benzoboroxoles: Novel Organoboron Heterocycles. Tetrahedron Lett. 1999, 40 (37), 6705-6708.

(12) Park, Y. H.; Ahn, H. R.; Canturk, B.; Jeon, S. I.; Lee, S.; Kang, H.; Molander, G. A.; Ham, J. A Facile One-pot Preparation of Potassium Hydroxyaryl- and (Hydroxyalkyl)aryltrifluoroborates. Org. Lett. 2008, 10 (6), 1215-1218.

(13) Ye, L.; Ding, D.; Feng, Y.; Xie, D.; Wu, P.; Guo, H.; Meng, Q.; Zhou, H. Convenient and Versatile Synthesis of Formyl-Substituted Benzoxaboroles. Tetrahedron 2009, 65, 8738-8744.

(14) Lulinski, S.; Madura, I.; Serwatowski, J.; Szatylowicz, H.; Zachara, J. A Tautomeric Equilibrium Between Functionalized 2- formylphenylboronic acids and Corresponding 1,3-Dihydro-1,3dihydroxybenzo $[\mathrm{c}][2,1]$ oxaboroles. New J. Chem. 2007, 31 (1), 144-154.

(15) Lin, S.; Song, C.-X.; Cai, G.-X.; Wang, W.-H.; Shi, Z.-J. Intra/ Intermolecular Direct Allylic Alkylation via Pd(II)-Catalyzed Allylic CH Activation. J. Am. Chem. Soc. 2008, 130 (39), 12901-12903.

(16) Molander, G. A.; Sandrock, D. L. Orthogonal Reactivity in Boryl-Substituted Organotrifluoroborate. J. Am. Chem. Soc. 2008, 130 (47), 15792-15793.

(17) Kowada, T.; Yamaguchi, S.; Fujinaga, H.; Ohe, K. Near-Infrared BODIPY Dyes Modulated with Spirofluorene Moieties. Tetrahedron 2011, 67 (17), 3105-3110.

(18) Brownlee, R. T. C.; Craik, D. J. Temperature-Dependence of F19 Chemical-Shifts in Substituted Benzyl Fluorides. Tetrahedron Lett. 1980, 21 (17), 1681-1684.

(19) Anet, F. A. L.; Yavari, I.; Ferguson, I. J.; Katritzky, A. R.; Moreno-Manas, M.; Robinson, M. J. T. The Conformational-Analysis of Saturated Heterocycles. N-Inversion in Hindered Piperidines. J. Chem. Soc., Chem. Commun. 1976, 11, 399-400.

(20) Gutowsky, H. S.; Holm, C. H. Rate Processes and Nuclear Magnetic Resonance Spectra. 2. Hindered Internal Rotation of Amides. J. Chem. Phys. 1956, 25 (6), 1228-1234.

(21) Furikado, Y.; Nagahata, T.; Okamoto, T.; Sugaya, T.; Iwatsuki, S.; Inamo, M.; Takagi, H. D.; Odani, A.; Ishihara, K. Universal Reaction Mechanism of Boronic Acids with Diols in Aqueous Solution: Kinetics and the Basic Concept of a Conditional Formation Constant. Chem. - Eur. J. 2014, 20, 13194-13202.

(22) Yamamoto, Y.; Matsumura, T.; Takao, N.; Yamagishi, H.; Takahashi, M.; Iwatsuki, S.; Ishihara, K. Fast Trigonal/Tetragonal Interconversion Followed by Slow Chelate-Ring Closure in the Complexation of Boronic Acids. Inorg. Chim. Acta 2005, 358 (12), 3355-3361. 